\title{
Interaction of suprathermal solar wind electron fluxes with sheared whistler waves: fan instability
}

\author{
C. Krafft ${ }^{1}$ and A. Volokitin ${ }^{2}$ \\ ${ }^{1}$ Laboratoire de Physique des Gaz et des Plasmas, Université Paris Sud, 91405 Orsay Cedex, France \\ ${ }^{2}$ Institute of Terrestrial Magnetism, Ionosphere and Radiowave Propagation, Academy of Sciences, Troitsk, Moscow Region, \\ 142190, Russia
}

Received: 13 September 2002 - Revised: 20 December 2002 - Accepted: 5 February 2003

\begin{abstract}
Several in situ measurements performed in the solar wind evidenced that solar type III radio bursts were sometimes associated with locally excited Langmuir waves, highenergy electron fluxes and low-frequency electrostatic and electromagnetic waves; moreover, in some cases, the simultaneous identification of energetic electron fluxes, Langmuir and whistler waves was performed. This paper shows how whistlers can be excited in the disturbed solar wind through the so-called "fan instability" by interacting with energetic electrons at the anomalous Doppler resonance. This instability process, which is driven by the anisotropy in the energetic electron velocity distribution along the ambient magnetic field, does not require any positive slope in the suprathermal electron tail and thus can account for physical situations where plateaued reduced electron velocity distributions were observed in solar wind plasmas in association with Langmuir and whistler waves. Owing to linear calculations of growth rates, we show that for disturbed solar wind conditions (that is, when suprathermal particle fluxes propagate along the ambient magnetic field), the fan instability can excite VLF waves (whistlers and lower hybrid waves) with characteristics close to those observed in space experiments.
\end{abstract}

Key words. Space plasma physics (waves and instabilities) - Radio Science (waves in plasma) - Solar physics, astrophysics and astronomy (radio emissions)

\section{Introduction}

Solar type III radio bursts are generated by energetic electron beams - sometimes associated with solar flares - which originate from the solar corona and travel along open magnetic lines toward the interplanetary space (Zaitsev et al., 1972, 1974; Melrose, 1974; Gurnett and Anderson, 1976). Several in situ measurements evidenced that these radio bursts were sometimes associated with locally excited Langmuir waves,

Correspondence to: C. Krafft

(Catherine.Krafft@lpgp.u-psud.fr) high-energy electron fluxes and low-frequency electrostatic and electromagnetic waves (Lin et al., 1981, 1986, 1998; Kellogg et al., 1992a, b; Stone et al., 1995; Reiner et al., 1992; Thejappa et al., 1995; Ergun et al., 1998; Thejappa and MacDowall, 1998; Moullard et al., 1998, 2001); moreover, the simultaneous identification of energetic electron fluxes, Langmuir and whistler waves was performed by some of the cited experiments. It is commonly believed that the impulsive solar electrons ejected from the corona develop a streaming anisotropy as the faster electrons catch up to the slower ones, which results in the appearance of a bump (or beam) in the tail of the electron velocity distribution. Langmuir waves are then supposed to be generated by a bump-in-tail instability which is saturated by the quasi-linear relaxation of the beam and finally leads to the flattening of the velocity distribution in the tail region (Ginzburg and Zheleznyakov, 1958). The Langmuir waves excited at the plasma frequency $\omega_{p}$ are then believed to be involved in nonlinear wave-wave interaction processes and to be converted in escaping radiation at the plasma frequency and its harmonic $2 \omega_{p}$, giving rise to strong radio emissions (e.g. Papadopoulos et al., 1974; Bardwell and Goldman, 1976; Smith et al., 1979). Even if several in situ observations confirm part of this scenario, many features governing the mechanisms of the appearance of these radio bursts remain, up until now, to be understood. Indeed, many questions remain to be solved concerning the generation mechanisms of each type of wave which participates in the production of the bursts, the processes that govern the high-energy fluxes' evolution and the role of nonlinear wavewave interactions. In this paper, our attention will be focused on the role of low-frequency waves as whistlers and lower hybrid waves in the solar wind and more specifically, in the generation of type III solar radio bursts.

Whistlers observed in the solar wind are usually believed to be generated by some instability caused by the distortion of the electron velocity distribution from the maxwellian one. Most probable instabilities are due to the anisotropy between the perpendicular and the parallel electronic temperatures (see, e.g. Mace, 1998; Gary and Cairns, 1999; Zhang et al., 
1999b), to the anisotropy of the parallel velocity distribution which appears through heat transfer (Forslund et al., 1972; Gary et al., 1975; Jie Zhao et al., 1996), or to the presence of suprathermal electron fluxes or beams (Kennel and Wong, 1967; Tokar et al., 1984; Ergun et al., 1993; Omelchenko et al., 1994). Whistlers can also be excited by nonlinear wavewave processes; let us cite, for example, the decay of a Langmuir wave into another Langmuir wave with the participation of whistlers and lower hybrid waves, as considered by Kuo and Lee (1989), Leyser (1991), Sawhney et al. (1996) and Sharma et al. (1998) for ionospheric and laboratory experiment conditions, and only by Abalde et al. (1998), Chian and Abalde (1999) and Luo et al. (2000) for solar wind plasma conditions.

During the solar III radio bursts observed by the Wind spacecraft (Ergun et al., 1998), locally enhanced Langmuir waves were observed in the solar wind in association with fluxes of high-energy solar impulsive electrons ranging from 2 to $12 \mathrm{keV}$, as well as with low-frequency electromagnetic and electrostatic emissions (whistlers, lower hybrid waves and ion acoustic waves). However, the measured electron reduced velocity distributions (that is, the total electron velocity distributions integrated on the perpendicular velocity) were rarely exhibiting bump-in-tail features and for most of the recorded data the distributions were marginally stable or plateaued during the appearance of the strong Langmuir emissions. Nevertheless, in the case of the ISEE-3 experiment (Lin et al., 1981), very clear and long-living bumps in the reduced electron distributions were detected, that is, strong positive slopes persisting for periods greater than $10 \mathrm{~min}$. This discrepancy between both experiments, maybe partly due to the solar activity during measurements, allow one to suppose that not only one, but several various physical phenomena should play and interfere together.

Moreover, such kinds of observations were also performed in other regions of the solar wind where the electron velocity distributions exhibit various peculiarities. Indeed, locally enhanced Langmuir waves were observed in the solar wind in association with high-energy electron fluxes and whistlers in the auroral ionosphere above $500 \mathrm{~km}$ altitude (Ergun et al., 1993; Muschietti et al., 1997). The following mechanism was proposed to describe one possible source of growth for VLF waves: after the reduced electron velocity distribution is plateaued by the Langmuir oscillations, it can however remain unstable to electrostatic whistlers with a finite perpendicular wave number that can interact at the Landau resonance with field-aligned electrons of energies ranging from $100 \mathrm{eV}$ to $3 \mathrm{keV}$. On another hand, reporting on lowfrequency magnetic field fluctuations that are enhanced behind interplanetary shocks, Corotini et al. (1982) presented indirect evidence that whistlers propagating at very large normal angles (above 70 degrees) and with wavelengths of the order of $c / \omega_{p}$ may be generated in the solar wind during disturbed times. Owing to some theoretical study, authors argue that these oblique whistlers may be excited by electron free energy, although no measurements of particle fluxes were available. Sentman et al. (1983) suggest that these whistlers may be driven by a non-maxwellian feature of the solar wind velocity distribution (pear-shaped structure) in disturbed times and may grow via Landau resonance with this free source. Such type of distribution is closely associated with the presence of obliquely propagating whistlers with a frequency of around $1 \mathrm{~Hz}$, which were often observed within the electron foreshock (Hoppe et al., 1982). Extremely intense parallel-propagating whistlers were also evidenced recently near the bow shock by the Geotail satellite, and were believed to be excited by electron beams with temperature anisotropy (Zhang et al., 1999b). The same mechanism of excitation is proposed to explain the observation of quasi-parallel propagating whistlers in the Earth's magnetotail (Zhang et al., 1999a) or the correlation which was evidenced in the plasma sheet boundary layer between broadband electrostatic noise around $10 \mathrm{kHz}$, energetic electrons around $1 \mathrm{keV}$ to tens of $\mathrm{keV}$ and whistler mode magnetic noise bursts below $178 \mathrm{~Hz}$ (Parks et al., 1984).

After this brief list of examples showing the presence of whistlers in the solar wind in various regions of the interplanetary space and of the near-Earth's environment, one can ask the fundamental following questions: what is the source of the whistlers observed in the solar wind and, more specifically, what is the source of the whistlers observed in association with solar bursts? Are whistlers and Langmuir waves coupled through nonlinear wave-wave interactions (Kennel et al., 1980) or are they excited simultaneously by the electron fluxes (Thejappa et al., 1995)? What influence do whistlers produce on the Langmuir turbulence, which is believed to be the main agent of the radio bursts? Do the whistlers play a role in the fact that the electron fluxes can propagate along very long distances from the solar corona to 1 AU before being plateaued, as shown by observations? Indeed, one can suppose that Langmuir excitation will lead to strong particle diffusion in velocity space and will not allow for the propagation of a coherent stream of electrons far from the Sun, but only along a few kilometers. Thus, in order to explain the observations, one has to find which mechanisms can stabilize the Langmuir instability so that waves will not grow enough to interact with the beam and destroy the bump; for example, it was proposed that nonlinear processes - as induced scattering, Langmuir backscatter, modulational instability, strong turbulence, electrostatic decay (Kaplan and Tsytovich, 1973; Papadopoulos et al., 1974; Smith et al., 1979) - could scatter the Langmuir waves out of the resonance with the beam in a time scale much shorter than the quasi-linear relaxation process and thus suppress it. Moreover, during the quasi-linear relaxation process, what is the influence of the parallel heating of the plasma bulk, as well as the modification of the temperature in the beam region, on the temperature anisotropy of the velocity distribution which is supposed to drive important instabilities? Clear answers to these various questions remain, up until now, to be provided.

In the physical scenario that we propose here, one assumes that after the quasi-linear relaxation of the bump in the electron parallel velocity distribution due to Langmuir turbulence, whistlers can be excited through the so-called 
"fan instability" by interacting with the energetic electrons at the anomalous Doppler resonance. This instability does not require any positive slope in the suprathermal electron tail and thus can account for physical situations where plateaued reduced electron velocity distributions were observed in association with Langmuir and whistler waves; in this case, whistlers can use the free energy from the beam that is not available for Langmuir waves. This instability process is driven by the anisotropy in the energetic electron velocity distribution along the ambient magnetic field (Shapiro and Shevchenko, 1968; Haber et al., 1978). It was first discussed in the frame of thermonuclear fusion by Kadomtsev and Pogutse (1967) and was namely shown to generate electron Bernstein modes in the magnetosphere (Volokitin and Lizunov, 1995) or lower hybrid waves in the ionosphere with electron currents (Atamanyuk and Volokitin, 2001). The threshold of this instability is overcome if the number of electrons giving energy to the wave interacting at the anomalous Doppler resonance exceeds the number of electrons taking energy from the wave at the Cherenkov and the normal Doppler resonances (Mikhailovskii, 1974; Omelchenko et al., 1994). Considering the nonlinear stage, this fan instability can be shown to saturate, owing to particle trapping and exchanges of energy between waves and particles, producing a bump in the tail of the parallel velocity distribution (Volokitin and Krafft, 2003). This bump can, in turn, excite waves through various mechanisms and influence noticeably the electron suprathermal tail evolution.

However, one could argue that such an instability process cannot excite waves easily due to the fact that the parallel (as well as the perpendicular) velocity distribution function of the solar wind hot electronic population has been shown to decrease as a power law and, thus, that the amount of particles at Landau resonance is many orders of magnitude larger than the particle flux present at the anomalous cyclotron resonant velocity, making it hard for the hot electrons in anomalous cyclotron resonance with waves to overcome the stabilizing effect of the more cold Landau resonant electrons. But we consider here suprathermal tails produced by any disturbances which can enhance the hot tail population. For example, as we explained above, after the rapidly growing Langmuir waves lead to a flatter distribution function in the region of the positive slope, the particles diffuse to lower velocity according to quasi-linear relaxation, feeding the suprathermal tail of the parallel distribution function with electrons which can interact at the anomalous cyclotron resonance with VLF waves.

Owing to linear calculations of growth rates, we demonstrate in this paper that, for disturbed solar wind conditions (that is, when suprathermal particles fluxes propagate along the ambient magnetic field), the fan instability can excite VLF waves (whistlers and lower hybrid waves) with characteristics close to those observed in the solar wind. Considering two of the examples cited above, that is the cases of auroral solar wind and type III solar radio burst plasma conditions, calculations show that oblique whistlers can be excited through the mechanism of fan instability at the same frequencies as those observed in the space experiments.

\section{Fan instability of sheared whistlers}

\subsection{Linear growth rate}

Sheared whistlers, or so-called electromagnetic lower hybrid waves, are oblique propagating whistlers whose frequencies $\omega$ are much lower than the electron gyrofrequency, $\omega \ll \omega_{c}$, and whose parallel wave numbers are much less than their perpendicular ones, $k_{z}^{2} \ll k_{\perp}^{2} \simeq k^{2}$. Their dispersion relation is

$$
\frac{\omega^{2}}{\omega_{p}^{2}}+\frac{\omega^{2}}{\omega_{c}^{2}}\left(1+\frac{\omega_{p}^{2}}{c^{2} k^{2}}\right)=\frac{\omega_{p i}^{2}}{\omega_{p}^{2}}+\frac{k_{z}^{2}}{k^{2}+\omega_{p}^{2} / c^{2}},
$$

which can be written for most of the typical solar wind conditions where $\omega_{p} \gg \omega_{c}$ as

$$
\frac{\omega^{2}}{\omega_{c}^{2}}\left(1+\frac{\omega_{p}^{2}}{c^{2} k^{2}}\right) \simeq \frac{\omega_{p i}^{2}}{\omega_{p}^{2}}+\frac{k_{z}^{2}}{k^{2}+\omega_{p}^{2} / c^{2}},
$$

where $\omega_{p}$ and $\omega_{p i}$ are the electron and ion plasma frequencies, respectively; $k$ is the modulus of the wave number $\boldsymbol{k}\left(\boldsymbol{k}_{\perp}, k_{z}\right)$. This relation can be easily obtained using the Maxwell equations in the $k$-space

$\left(k^{2} \delta_{i j}-k_{i} k_{j}\right) E_{j}-\left(\frac{\omega}{c}\right)^{2} \varepsilon_{i j} E_{j}=0$,

where $E_{j}$ is the $j$-component of the electric field, as well as the components $\varepsilon_{i j}$ of the dielectric tensor in the cold plasma approximation

$$
\begin{aligned}
& \varepsilon_{x x}=\varepsilon_{y y} \equiv \varepsilon_{\perp} \simeq 1-\frac{\omega_{p}^{2}}{\omega^{2}-\omega_{c}^{2}}-\frac{\omega_{p i}^{2}}{\omega^{2}} \\
& \varepsilon_{x y}=-\varepsilon_{y x} \equiv i q \simeq \frac{\omega_{c} \omega_{p}^{2}}{\omega\left(\omega^{2}-\omega_{c}^{2}\right)}, \varepsilon_{z z} \equiv \varepsilon_{\|} \simeq 1-\frac{\omega_{p}^{2}}{\omega^{2}},
\end{aligned}
$$

where the small components $\varepsilon_{x z}$ and $\varepsilon_{y z}$ can be neglected. Then Eq. (3) can be presented as $\left(k_{y}=0\right)$

$$
\frac{k_{z}^{2} c^{2}}{\omega^{2}}-\varepsilon_{\perp}-\frac{q^{2}}{\frac{k^{2} c^{2}}{\omega^{2}}-\varepsilon_{\perp}} \simeq \frac{1}{\frac{k_{x}^{2} c^{2}}{\omega^{2}}-\varepsilon_{\|}} \frac{k_{x}^{2} k_{z}^{2} c^{4}}{\omega^{4}},
$$

and, neglecting the small terms proportional to $\omega^{2} / \omega_{p}^{2}$ and $\omega^{2} / \omega_{c}^{2}$, one obtains

$$
\frac{k_{z}^{2} c^{2}}{\omega^{2}}+\frac{\omega_{p i}^{2}}{\omega^{2}}-\left(1+\frac{\omega_{p}^{2}}{\omega_{c}^{2}}\right)-\frac{\omega_{p}^{4} / \omega_{c}^{2}}{k^{2} c^{2}} \simeq \frac{k_{x}^{2} k_{z}^{2} c^{4}}{\omega^{2}\left(k_{x}^{2} c^{2}+\omega_{p}^{2}\right)},(6)
$$

which leads to Eq. (1) when assuming that $k_{z}^{2} \ll k_{\perp}^{2}$.

The complex electric field $\boldsymbol{E}$ of the sheared whistler can be expressed in a Fourier series as

$$
\boldsymbol{E}=\sum_{\boldsymbol{k}} \boldsymbol{E}_{\boldsymbol{k}} \exp (-i \omega t+i \boldsymbol{k} \cdot \boldsymbol{r}), \quad \boldsymbol{E}_{\boldsymbol{k}}=\boldsymbol{E}_{\perp \boldsymbol{k}}+z E_{z \boldsymbol{k}},
$$


with (Volokitin et al., 1995)

$\boldsymbol{E}_{\perp \boldsymbol{k}}=-\nabla_{\perp} \varphi_{\boldsymbol{k}}=-i \boldsymbol{k}_{\perp} \varphi_{\boldsymbol{k}}$,

$E_{z \boldsymbol{k}}=-i k_{z} \varphi_{\boldsymbol{k}}+i \frac{\omega}{c} A_{z \boldsymbol{k}}=-i k_{z} \varphi_{\boldsymbol{k}} \frac{c^{2} k^{2}}{\omega_{p}^{2}+c^{2} k^{2}}$,

and

$\boldsymbol{A}_{\boldsymbol{k}} \simeq z A_{z k}, \quad \boldsymbol{A}_{\perp k} \simeq 0$.

$\varphi_{\boldsymbol{k}}$ and $\boldsymbol{A}_{\boldsymbol{k}}$ are the scalar and the vector potentials corresponding to the wave $(\omega, \boldsymbol{k})$ and $\boldsymbol{z}$ is the unit vector along the ambient magnetic field $\boldsymbol{B}_{0}$. The energy density of the sheared whistlers is

$W_{\boldsymbol{k}}=\left\langle\frac{\boldsymbol{B}^{2}}{8 \pi}+\frac{\boldsymbol{E}^{2}}{8 \pi}+\frac{m_{e} n_{e} \boldsymbol{v}_{e}^{2}}{2}+\frac{m_{i} n_{i} \boldsymbol{v}_{i}^{2}}{2}\right\rangle$,

where $n_{e}$ and $n_{i}$ are the electronic and ionic densities, $n_{e} \simeq$ $n_{i} \simeq n_{0} ; \boldsymbol{B}$ is the magnetic wave field; $\boldsymbol{v}_{e}$ and $\boldsymbol{v}_{i}$ are the electron and ion velocities. After some calculations, one obtains

$W_{\boldsymbol{k}} \simeq \frac{k^{2}\left|\varphi_{\boldsymbol{k}}\right|^{2}}{2 \pi} \frac{\omega_{p}^{2}}{\omega_{c}^{2}}\left[1+\frac{\omega_{c}^{2}}{2 \omega_{p}^{2}}+\frac{\omega_{p}^{2}}{c^{2} k^{2}}\right]$.

The total instability growth rate $\gamma$ can be calculated considering the exchange of wave energy with ions and electrons

$$
\begin{gathered}
\frac{\partial}{\partial t} W_{\boldsymbol{k}}=-\left\langle\boldsymbol{j}_{e} \cdot \boldsymbol{E}\right\rangle-\left\langle\boldsymbol{j}_{i} \cdot \boldsymbol{E}\right\rangle \\
=2\left(\gamma_{e}+\gamma_{i}\right) W_{\boldsymbol{k}} \equiv 2 \gamma W_{\boldsymbol{k}},
\end{gathered}
$$

where $\boldsymbol{j}_{i}$ and $\boldsymbol{j}_{e}$ are the ionic and electronic current densities. The average work produced by the wave field $\boldsymbol{E}\left(E_{x}, E_{y}, E_{z}\right)$ on the electrons is

$\left\langle\boldsymbol{j}_{e} \cdot \boldsymbol{E}\right\rangle$

$=-e n_{e} \iiint\left(v_{x} E_{x}^{*}+v_{y} E_{y}^{*}+v_{z} E_{z}^{*}\right) \delta f_{e} d^{3} v+c . c .$,

where $\left(v_{x}, v_{y}, v_{z}\right)$ are the cartesian coordinates of the electron velocity $\boldsymbol{v}$, and $\delta f_{e}$ is the perturbation of the electron distribution function $f_{e}$

$f_{e}=f_{0}+\delta f_{e}$,

$\delta f_{e}=\sum_{\boldsymbol{k}} \delta f_{\boldsymbol{k}}\left(v_{z}, v_{\perp}, \theta\right) \exp (-i \omega t+i \boldsymbol{k} \cdot \boldsymbol{r})$,

where $\theta$ is the azimuthal angle; $v_{z}$ and $v_{\perp}$ are the parallel and the perpendicular velocities, respectively $\left(v_{x}=\right.$ $\left.v_{\perp} \cos \theta, v_{y}=v_{\perp} \sin \theta\right)$. Then, the Vlasov equation leads to the expression

$\frac{\partial}{\partial \theta} \delta f_{k}+g_{1 k}(\theta) \delta f_{k}=g_{2 k}(\theta)$

where

$g_{1 \boldsymbol{k}}(\theta)=i \frac{\omega}{\omega_{c}}\left(\frac{\boldsymbol{k} \cdot \boldsymbol{v}}{\omega}-1\right)$,

$g_{2 \boldsymbol{k}}(\theta)=\frac{e}{m_{e} c}\left[\left(1-\frac{\boldsymbol{k} \cdot \boldsymbol{v}}{\omega}\right) \boldsymbol{E}_{\boldsymbol{k}}+\left(\frac{\boldsymbol{E}_{\boldsymbol{k}} \cdot \boldsymbol{v}}{\omega}\right) \boldsymbol{k}\right] \cdot \frac{\partial f_{0}}{\partial \boldsymbol{v}}$
After some calculations, Eq. (15) can be written as

$$
\begin{gathered}
\frac{\partial}{\partial \theta} \delta f_{\boldsymbol{k}}+i\left(\frac{k_{z} v_{z}-\omega}{\omega_{c}}+\frac{k_{\perp} v_{\perp}}{\omega_{c}} \cos \theta\right) \delta f_{\boldsymbol{k}} \\
=\frac{-i e \varphi_{\boldsymbol{k}}}{m_{e} \omega_{c}}\left[\frac{c^{2} k^{2} k_{z}}{\omega_{p}^{2}+c^{2} k^{2}} \frac{\partial f_{0}}{\partial v_{z}}\right. \\
\left.+k_{\perp} \cos \theta\left(\frac{\partial f_{0}}{\partial v_{\perp}}-\frac{\omega_{p}^{2}}{\omega_{p}^{2}+c^{2} k^{2}} \frac{k_{z}}{\omega} \Lambda f_{0}\right)\right],
\end{gathered}
$$

where

$\Lambda f_{0}\left(v_{z}, v_{\perp}\right) \equiv v_{z} \frac{\partial f_{0}}{\partial v_{\perp}}-v_{\perp} \frac{\partial f_{0}}{\partial v_{z}}$.

Solving Eq. (18) leads to

$$
\begin{aligned}
\delta f_{\boldsymbol{k}} & =\exp \left(-\int^{\theta} g_{1 \boldsymbol{k}}\left(\theta^{\prime}\right) d \theta^{\prime}\right) \\
& \left\{\int^{\theta} g_{2 \boldsymbol{k}}\left(\theta^{\prime}\right) \exp \left(\int^{\theta^{\prime}} g_{1 \boldsymbol{k}}\left(\theta^{\prime \prime}\right) d \theta^{\prime \prime}\right) d \theta^{\prime}+C\right\},
\end{aligned}
$$

where the constant $C$ vanishes, owing to the periodicity condition $\delta f_{k}(\theta)=\delta f_{k}(\theta+2 \pi)$. Then, one obtains

$$
\begin{aligned}
\delta f_{\boldsymbol{k}} & =\frac{-i e \varphi_{\boldsymbol{k}}}{m_{e} \omega_{c}} \int^{\theta} d \theta^{\prime} \\
& \exp \left(i \int_{\theta^{\prime}}^{\theta}\left(\frac{k_{z} v_{z}-\omega}{\omega_{c}}+\frac{k_{\perp} v_{\perp}}{\omega_{c}} \cos \theta^{\prime \prime}\right) d \theta^{\prime \prime}\right) \\
& \times\left[\frac{c^{2} k^{2} k_{z}}{\omega_{p}^{2}+c^{2} k^{2}} \frac{\partial f_{0}}{\partial v_{z}}\right. \\
& \left.+k_{\perp} \cos \theta^{\prime}\left(\frac{\partial f_{0}}{\partial v_{\perp}}-\frac{\omega_{p}^{2}}{\omega_{p}^{2}+c^{2} k^{2}} \frac{k_{z}}{\omega} \Lambda f_{0}\right)\right],
\end{aligned}
$$

and, defining

$$
\begin{aligned}
& \alpha=\frac{c^{2} k^{2}}{\omega_{p}^{2}+c^{2} k^{2}}, \quad \beta=1-\alpha \\
& \quad G\left(v_{z}, v_{\perp}\right)=\left[\alpha k_{z} \frac{\partial f_{0}}{\partial v_{z}}+\frac{n \omega_{c}}{v_{\perp}}\left(\frac{\partial f_{0}}{\partial v_{\perp}}-\beta \frac{k_{z}}{\omega} \Lambda f_{0}\right)\right]
\end{aligned}
$$

one obtains

$$
\begin{gathered}
\delta f_{\boldsymbol{k}}=-\frac{e \varphi_{\boldsymbol{k}}}{m_{e}} \sum_{n, m=-\infty}^{\infty} \frac{G\left(v_{z}, v_{\perp}\right) e^{i(n-m) \theta}}{k_{z} v_{z}-\omega+m \omega_{c}} \\
J_{n}\left(\frac{k_{\perp} v_{\perp}}{\omega_{c}}\right) J_{m}\left(\frac{k_{\perp} v_{\perp}}{\omega_{c}}\right),
\end{gathered}
$$

where $J_{n}$ is the Bessel function of order $n$. Then, the work (Eq. 13) can be expressed as follows

$\left\langle\boldsymbol{j}_{e} \cdot \boldsymbol{E}\right\rangle=-e n_{e} \int\left(v_{z} E_{z}^{*}+v_{\perp} E_{\perp}^{*}\right) \delta f_{e} d^{3} \boldsymbol{v}+$ c.c. 


$$
\begin{aligned}
=- & \text { ien } \int_{0}^{2 \pi} d \theta \int_{-\infty}^{\infty} d v_{z} \int_{0}^{\infty} v_{\perp} d v_{\perp} \\
& \left(\alpha v_{z} k_{z}+k_{\perp} v_{\perp} \cos \theta\right) \varphi_{\boldsymbol{k}}^{*} \delta f_{\boldsymbol{k}}+c . c . \\
=- & 4 \pi \frac{e^{2} n_{e}\left|\varphi_{\boldsymbol{k}}\right|^{2}}{m_{e}} \operatorname{Im}\left\{\sum_{n=-\infty}^{\infty} \int_{-\infty}^{\infty} \int_{0}^{\infty} \frac{d v_{z} v_{\perp} d v_{\perp}}{k_{z} v_{z}-\omega+n \omega_{c}}\right. \\
& \left.J_{n}^{2}\left(\frac{k_{\perp} v_{\perp}}{\omega_{c}}\right)\left(\alpha v_{z} k_{z}+n \omega_{c}\right) G\left(v_{z}, v_{\perp}\right)\right\}
\end{aligned}
$$

Using the Plemelj formula with $\omega=\omega_{r}+i \gamma$

$$
\begin{aligned}
& \lim _{\gamma \rightarrow \mathrm{O}^{+}} \int \frac{1}{\omega_{r}-k_{z} v_{z}-n \omega_{c}+i \gamma} \\
& \quad=\mathcal{P}\left(\frac{1}{\omega_{r}-k_{z} v_{z}-n \omega_{c}}\right)-i \pi \delta\left(\omega_{r}-k_{z} v_{z}-n \omega_{c}\right),
\end{aligned}
$$

we obtain the growth rate associated with the electrons

$$
\begin{aligned}
\gamma_{e}= & -\frac{\left\langle\boldsymbol{j}_{e} \cdot \boldsymbol{E}\right\rangle}{2 W_{\boldsymbol{k}}}=\frac{\left|\varphi_{\boldsymbol{k}}\right|^{2}}{W_{\boldsymbol{k}}} \frac{2 \pi^{2} e^{2} n_{e}}{m_{e}} \\
& \sum_{n=-\infty}^{\infty} \int_{-\infty}^{\infty} \int_{0}^{\infty} d v_{z} v_{\perp} d v_{\perp} J_{n}^{2}\left(\frac{k_{\perp} v_{\perp}}{\omega_{c}}\right) \\
& \left(\alpha v_{z} k_{z}+n \omega_{c}\right) G\left(v_{z}, v_{\perp}\right) \delta\left(\omega_{r}-k_{z} v_{z}-n \omega_{c}\right) .
\end{aligned}
$$

Then, noting $\omega$ instead of $\omega_{r}$, one finally obtains

$$
\begin{gathered}
\frac{\gamma_{e}}{\omega_{c}} \simeq \frac{\pi^{2} \omega \omega_{c} \operatorname{sign}\left(k_{z}\right)}{k^{2}\left(1+\frac{\omega_{c}^{2}}{2 \omega_{p}^{2}}+\frac{\omega_{p}^{2}}{c^{2} k^{2}}\right)} \sum_{n=-\infty}^{\infty}\left(\alpha+n(1-\alpha) \frac{\omega_{c}}{\omega}\right)^{2} \\
\int_{0}^{\infty} v_{\perp} d v_{\perp} J_{n}^{2}\left(\frac{k_{\perp} v_{\perp}}{\omega_{c}}\right)\left[\frac{\partial f_{0}}{\partial v_{z}}+\frac{n \omega_{c}}{k_{z} v_{\perp}} \frac{\partial f_{0}}{\partial v_{\perp}}\right]_{v_{z}=v_{z n}},
\end{gathered}
$$

where the resonant velocity is

$v_{z n}=\frac{\omega-n \omega_{c}}{k_{z}}$

The growth rate associated with the unmagnetized ions can be calculated by taking into account the damping of the wave at the Landau resonance

$\gamma_{i} \simeq-\frac{\operatorname{Im} \varepsilon}{\partial \operatorname{Re} \varepsilon / \partial \omega}$

where $\varepsilon(\omega, k)$ is the dielectric function. For a maxwellian ion velocity distribution

$f_{i}\left(v_{z}, v_{\perp}\right)=\left(\frac{1}{\pi}\right)^{3 / 2} v_{T_{i}}^{-3} \exp \left(-v^{2} / v_{T_{i}}^{2}\right), v_{T_{i}}^{2}=\frac{2 T_{i}}{m_{i}}$,

where $T_{i}$ and $v_{T_{i}}$ are the temperature and the thermal velocity of the ions, one can use the electrostatic limit where

$$
\begin{aligned}
\operatorname{Im} \varepsilon & \simeq \frac{4 \pi n_{0} e^{2}}{k^{2} T_{i}} \frac{\sqrt{\pi} \omega}{v_{T_{i}}|k|} \exp \left(-\frac{\omega^{2}}{k^{2} v_{T_{i}}^{2}}\right), \\
W_{\boldsymbol{k}} & =\omega \frac{\partial \operatorname{Re} \varepsilon}{\partial \omega} \frac{|\boldsymbol{E}|^{2}}{8 \pi} .
\end{aligned}
$$

The normalized ion growth rate for sheared whistlers is then given by

$$
\begin{aligned}
\frac{\gamma_{i}}{\omega_{c}} \simeq-\frac{\sqrt{\pi} \omega}{4} \frac{\omega \omega_{c}}{\left[1+\frac{\omega_{c}^{2}}{2 \omega_{p}^{2}}+\frac{\omega_{p}^{2}}{c^{2} k^{2}}\right] \omega_{p}^{2}} \frac{\omega_{p i}^{2}}{v_{T_{i}}^{3}|k| k^{2}} \\
\quad \exp \left(-\frac{\omega^{2}}{k^{2} v_{T_{i}}^{2}}\right) .
\end{aligned}
$$

\subsection{Expressions with reduced distribution functions}

Let us define the function $F_{n}\left(v_{z}\right)$ as the reduced electron velocity distribution

$$
F_{n}\left(v_{z}\right)=2 \pi \int_{0}^{\infty} v_{\perp} J_{n}^{2}\left(\frac{k_{\perp} v_{\perp}}{\omega_{c}}\right) f_{0}\left(v_{z}, v_{\perp}\right) d v_{\perp},
$$

so that

$$
\begin{gathered}
2 \pi \int_{0}^{\infty} v_{\perp} d v_{\perp} J_{n}^{2}\left(\frac{k_{\perp} v_{\perp}}{\omega_{c}}\right)\left[\frac{\partial f_{0}}{\partial v_{z}}+\frac{n \omega_{c}}{k_{z} v_{\perp}} \frac{\partial f_{0}}{\partial v_{\perp}}\right]_{v_{z}=v_{z n}} \\
=\frac{\partial F_{n}}{\partial v_{z}}\left(v_{z n}\right)-\frac{k_{\perp}^{2}}{2 \omega_{c} k_{z}}\left[F_{n-1}\left(v_{z n}\right)-F_{n+1}\left(v_{z n}\right)\right]
\end{gathered}
$$

Then one can write using Eq. (29) that

$$
\begin{gathered}
\frac{\gamma_{e}}{\omega_{c}} \simeq \frac{\pi^{2} \omega \omega_{c} \operatorname{sign}\left(k_{z}\right)}{2 \pi k^{2}\left(1+\frac{\omega_{c}^{2}}{2 \omega_{p}^{2}}+\frac{\omega_{p}^{2}}{c^{2} k^{2}}\right)} \sum_{n=-\infty}^{\infty}\left(\alpha+n(1-\alpha) \frac{\omega_{c}}{\omega}\right)^{2} \\
\left(\frac{\partial F_{n}}{\partial v_{z}}\left(v_{z n}\right)-\frac{k_{\perp}^{2}}{2 \omega_{c} k_{z}}\left[F_{n-1}\left(v_{z n}\right)-F_{n+1}\left(v_{z n}\right)\right]\right)
\end{gathered}
$$

Supposing further that $f_{0}\left(v_{z}, v_{\perp}\right)=f_{z}\left(v_{z}\right) f_{\perp}\left(v_{\perp}\right)$ and $k_{\perp} v_{\perp} / \omega_{c} \leq 1$, we have

$J_{0}\left(\frac{k_{\perp} v_{\perp}}{\omega_{c}}\right) \simeq 1, J_{1}\left(\frac{k_{\perp} v_{\perp}}{\omega_{c}}\right) \simeq \frac{k_{\perp} v_{\perp}}{2 \omega_{c}} \gg J_{2}\left(\frac{k_{\perp} v_{\perp}}{\omega_{c}}\right)$,

and, then, using the normalization of $f_{\perp}\left(v_{\perp}\right)$, one obtains

$F_{0}\left(v_{z n}\right) \simeq 2 \pi f_{z}\left(v_{z n}\right) \int_{0}^{\infty} v_{\perp} f_{\perp}\left(v_{\perp}\right) d v_{\perp}=f_{z}\left(v_{z n}\right)$

and

$$
\begin{aligned}
& F_{1}\left(v_{z n}\right)=F_{-1}\left(v_{z n}\right) \simeq 2 \pi f_{z}\left(v_{z n}\right) \int_{0}^{\infty} \\
& v_{\perp} f_{\perp}\left(v_{\perp}\right)\left(\frac{k_{\perp} v_{\perp}}{2 \omega_{c}}\right)^{2} d v_{\perp}=\frac{k_{\perp}^{2}\left\langle v_{\perp}^{2}\right\rangle}{4 \omega_{c}^{2}} f_{z}\left(v_{z n}\right) .
\end{aligned}
$$

Thus, taking into account only the main resonances $n=$ $0, \pm 1$, one obtains the total normalized growth rate of the sheared whistlers in the form

$$
\begin{aligned}
& \frac{\gamma}{\omega_{c}} \simeq \frac{\pi \omega \omega_{c} \operatorname{sign}\left(k_{z}\right)}{2 k^{2}\left(1+\frac{\omega_{c}^{2}}{2 \omega_{p}^{2}}+\frac{\omega_{p}^{2}}{c^{2} k^{2}}\right)}\left[\alpha^{2} \frac{\partial f_{z}}{\partial v_{z}}\left(\frac{\omega}{k_{z}}\right)+\frac{k_{\perp}^{2}\left\langle v_{\perp}^{2}\right\rangle}{4 \omega_{c}^{2}}\right. \\
& \left\{\alpha_{+}^{2} \frac{\partial f_{z}}{\partial v_{z}}\left(\frac{\omega-\omega_{c}}{k_{z}}\right)+\alpha_{-}^{2} \frac{\partial f_{z}}{\partial v_{z}}\left(\frac{\omega+\omega_{c}}{k_{z}}\right)\right\}
\end{aligned}
$$




$$
\begin{aligned}
& -\frac{k_{\perp}^{2}}{2 \omega_{c} k_{z}}\left[\alpha_{+}^{2} f_{z}\left(\frac{\omega-\omega_{c}}{k_{z}}\right)-\alpha_{-}^{2} f_{z}\left(\frac{\omega+\omega_{c}}{k_{z}}\right)\right] \\
& -\frac{\sqrt{\pi} \omega}{4} \frac{\omega \omega_{c}}{\left[1+\frac{\omega_{c}^{2}}{2 \omega_{p}^{2}}+\frac{\omega_{p}^{2}}{c^{2} k^{2}}\right] \omega_{p}^{2}} \frac{\omega_{p i}^{2}}{v_{T_{i}}^{3}|k| k^{2}} \exp \left(-\frac{\omega^{2}}{k^{2} v_{T_{i}}^{2}}\right),
\end{aligned}
$$

with

$$
\left(\alpha+\beta \frac{\omega_{c}}{\omega}\right)^{2}=\alpha_{+}^{2}, \quad\left(\alpha-\beta \frac{\omega_{c}}{\omega}\right)^{2}=\alpha_{-}^{2} .
$$

If the parallel velocity distribution function is a maxwellian (with no suprathermal tail), that is $f_{z}=f_{M}$, it is well known that no instability can develop and that the corresponding electron growth rate $\gamma_{e}=\gamma_{e M}$ is thus negative (even if some terms in the expression (41) are positive). Let us now superpose to this maxwellian a suprathermal tail with a negative or constant slope in the region where the parallel velocity $v_{z}$ is positive. The electron growth rate $\gamma_{e}$ of the resulting distribution $f_{M}+f_{T}$ is the sum of the growth rates $\gamma_{e M}$ and $\gamma_{e T}$ due to each distribution. The terms contributing to the growth rate $\gamma_{e T}$ for the tail distribution (with no maxwellian) are the following: $\operatorname{sign}\left(k_{z}\right) \frac{k_{\perp}^{2}}{2 \omega_{c} k_{z}} \alpha_{-}^{2} f_{z}\left(\frac{\omega+\omega_{c}}{k_{z}}\right)$ is obviously a positive term, $\operatorname{sign}\left(k_{z}\right) \alpha_{-}^{2} \frac{\partial f_{z}}{\partial v_{z}}\left(\frac{\omega+\omega_{c}}{k_{z}}\right)$ is negative or zero (tail with negative or constant slope), the terms $\operatorname{sign}\left(k_{z}\right) \frac{k_{\perp}^{2}\left\langle v_{\perp}^{2}\right\rangle}{4 \omega_{c}^{2}} \alpha_{+}^{2} \frac{\partial f_{z}}{\partial v_{z}}\left(\frac{\omega-\omega_{c}}{k_{z}}\right)$ and $-\operatorname{sign}\left(k_{z}\right) \frac{k_{\perp}^{2}}{2 \omega_{c} k_{z}} \alpha_{+}^{2} f_{z}\left(\frac{\omega-\omega_{c}}{k_{z}}\right)$ give no contributions because $f_{T}=0$ for negative velocities (we have supposed that $k_{z}>0$ and thus we consider all the range of positive and negative velocities; here, $v_{z}=\frac{\omega-\omega_{c}}{k_{z}}<0$ ), and $\operatorname{sign}\left(k_{z}\right) \alpha^{2} \frac{\partial f_{z}}{\partial v_{z}}\left(\frac{\omega}{k_{z}}\right)$ is negative or zero. Thus, the only positive contribution to the growth rate when the distribution function is constituted by a maxwellian bulk and a suprathermal tail comes from the term $\operatorname{sign}\left(k_{z}\right) \frac{k_{\perp}^{2}}{2 \omega_{c} k_{z}} \alpha_{-}^{2} f_{z}\left(\frac{\omega+\omega_{c}}{k_{z}}\right)>$ 0 . This means that a necessary condition for the fan instability to develop is that the amount of particles in the velocity region of the anomalous Doppler resonance is enough to balance the negative contributions provided by the other terms.

In the limit of lower hybrid waves, one has $\alpha=1$ and

$$
W_{k} \simeq \frac{k^{2}\left|\varphi_{\boldsymbol{k}}\right|^{2}}{2 \pi} \frac{\omega_{p}^{2}}{\omega_{c}^{2}}\left[1+\frac{\omega_{c}^{2}}{\omega_{p}^{2}}\right],
$$

so that the normalized growth rate associated with the electrons in Eq. (41) can be simplified as

$$
\begin{aligned}
\frac{\gamma_{e}}{\omega_{c}} & \simeq \frac{\pi \omega \omega_{c} \operatorname{sign}\left(k_{z}\right)}{2 k^{2}\left(1+\frac{\omega_{c}^{2}}{\omega_{p}^{2}}\right)}\left\{\frac{\partial f_{z}}{\partial v_{z}}\left(\frac{\omega}{k_{z}}\right)+\frac{k_{\perp}^{2}\left\langle v_{\perp}^{2}\right\rangle}{4 \omega_{c}^{2}}\right. \\
& \times\left[\frac{\partial f_{z}}{\partial v_{z}}\left(\frac{\omega-\omega_{c}}{k_{z}}\right)+\frac{\partial f_{z}}{\partial v_{z}}\left(\frac{\omega+\omega_{c}}{k_{z}}\right)\right] \\
& \left.-\frac{k_{\perp}^{2}}{2 \omega_{c} k_{z}}\left[f_{z}\left(\frac{\omega-\omega_{c}}{k_{z}}\right)-f_{z}\left(\frac{\omega+\omega_{c}}{k_{z}}\right)\right]\right\} .
\end{aligned}
$$

\section{Fan instability in the solar wind}

Let us now estimate if sheared whistler waves or lower hybrid waves can be excited in the solar wind by the mechanism of fan instability, considering, for example, typical solar wind parameters recorded in situ by space experiments during the observations of solar type III radio bursts (Thejappa et al., 1995; Thejappa and MacDowall, 1998; Ergun et al., 1998) or of dispersive bursts of field-aligned electron fluxes in the Earth's auroral region (Ergun et al., 1993). For the first case, one chooses measurements from Ergun et al. (1998) and Thejappa and MacDowall (1998)

$$
\begin{aligned}
& \frac{\omega_{p}}{\omega_{c}} \simeq 167, \frac{\omega_{p}}{2 \pi} \simeq 25 \mathrm{kHz}, \frac{\omega_{c}}{2 \pi} \simeq 150 \mathrm{~Hz} \\
& 2-3 \omega_{l h} \lesssim \omega_{r} \lesssim \frac{\omega_{c}}{2}, \\
& E_{b} \simeq 2-12 \mathrm{keV}, T_{e} \simeq 10 \mathrm{eV}, \frac{T_{e}}{T_{i}} \simeq 2, \\
& 10^{-3} \lesssim k_{\perp} \lesssim 210^{-3} \mathrm{~m}^{-1},
\end{aligned}
$$

and from Ergun et al. (1998) and Thejappa et al. (1995)

$$
\begin{aligned}
& \frac{\omega_{p}}{\omega_{c}} \simeq 130, \frac{\omega_{p}}{2 \pi} \simeq 13 \mathrm{kHz}, \frac{\omega_{c}}{2 \pi} \simeq 100 \mathrm{~Hz} \\
& 2-3 \omega_{l h} \lesssim \omega_{r} \lesssim \frac{\omega_{c}}{2}, \\
& E_{b} \simeq 2-12 \mathrm{keV}, T_{e} \simeq 15 \mathrm{eV}, \frac{T_{e}}{T_{i}} \simeq 4, \\
& 10^{-4} \lesssim k_{\perp} \lesssim 510^{-4} \mathrm{~m}^{-1},
\end{aligned}
$$

where $\omega_{l h}$ is the lower hybrid frequency and $E_{b}$ is the beam energy domain resonant with the Langmuir waves detected along with the whistlers; $T_{e}$ and $T_{i}$ are the electron and ion temperatures; $k_{\perp}$ and $\omega_{r}$ are the perpendicular wave vector and the frequency of the waves identified as whistlers, respectively. Note that the two chosen samples of parameters (45)-(46) are rather similar, but the corresponding perpendicular wave numbers are different by one order of magnitude.

On another hand, the auroral events discussed in Ergun et al. (1993) are typical of the following physical conditions:

$$
\begin{gathered}
\frac{\omega_{p}}{\omega_{c}} \simeq 0.8, \frac{\omega_{p}}{2 \pi} \simeq 0.9 \mathrm{MHz}, \frac{\omega_{c}}{2 \pi} \simeq 1.15 \mathrm{MHz}, \\
2-3 \omega_{l h} \lesssim \omega_{r} \lesssim \frac{\omega_{c}}{2}, T_{e} \simeq 0.2 \mathrm{eV}, \\
0.025 \lesssim k_{\perp} \lesssim 0.1 \mathrm{~m}^{-1} .
\end{gathered}
$$

In the three cases (45)-(47), "oblique propagating" whistlers have been identified and thus one can suppose that their parallel wave vectors verify $\left|k_{z}\right| \ll\left|k_{\perp}\right|$. However, let us mention that for Eq. (47), the whistler emissions did not appear to have been directly correlated with enhanced fluxes of lowenergy electrons observed between $100 \mathrm{eV}$ and $3 \mathrm{keV}$.

The physical conditions (45)-(47) have been used to calculate numerically the linear growth rates of sheared whistlers excited in the solar wind by the fan instability. Note that our aim here is not to consider very complex electron 
(a) $f_{0}\left(v_{z}, v_{\perp}\right)$



(b) $\omega_{r} / \omega_{c}$
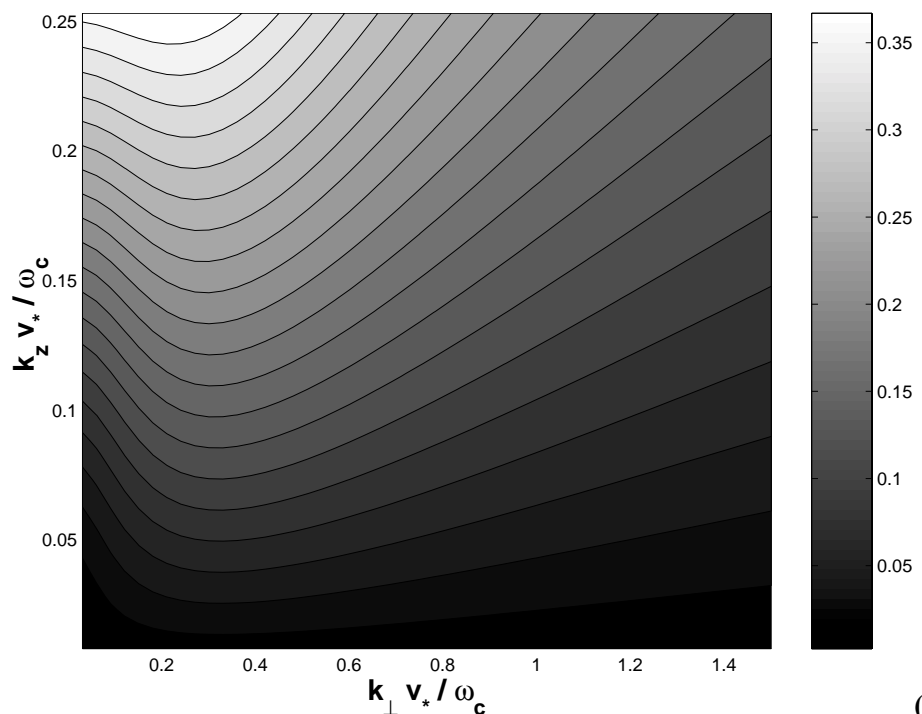

(c) $\gamma / \omega_{c}$

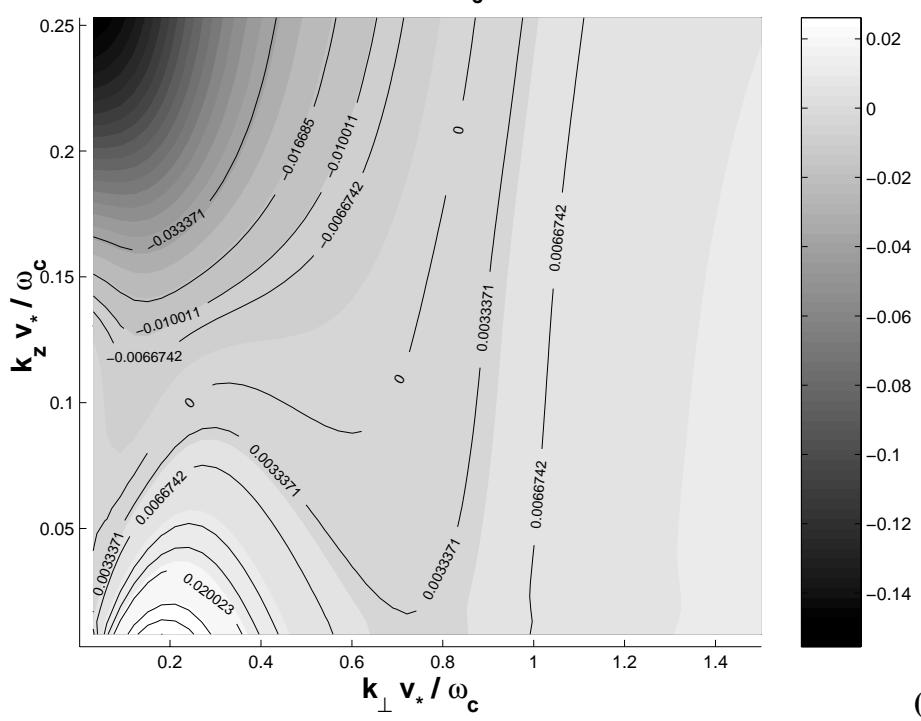

(c)

Fig. 1. Numerical calculations for solar wind plasma conditions: contour lines of constant level of (a) the electron velocity distribution $f_{0}\left(v, v_{\perp}\right)$ (in arbitrary units) as a function of the normalized parallel and perpendicular velocities $v_{z} / v_{*}$ and $v_{\perp} / v_{*}$, (b) the normalized frequency $\omega_{r} / \omega_{c}$ as a function of the normalized parallel and perpendicular wave numbers $k_{z} v_{*} / \omega_{c}$ and $k_{\perp} v_{*} / \omega_{c}$, and (c) the normalized growth rate $\gamma / \omega_{c}$ as a function of $k_{z} v_{*} / \omega_{c}$ and $k_{\perp} v_{*} / \omega_{c}$. The solar wind parameters are: $\omega_{p} / \omega_{c} \simeq 167, \omega_{c} / 2 \pi \simeq 150 \mathrm{~Hz}, \omega_{p} / 2 \pi \simeq$ $25 \mathrm{kHz}, T_{e} \simeq 10 \mathrm{eV}, T_{e} / T_{i} \simeq 2$. For (c), the values of the calculated growth rates are indicated on the picture. 
velocity distributions but to show that oblique propagating whistlers can be excited through the fan instability when a suprathermal population of solar wind electrons is present along the magnetic field lines. Thus, we simply model the total electronic population by a maxwellian with a temperature $T_{e}$ for the cold core and by a suprathermal tail decreasing as a power law for the hot population; indeed, the most important point here is to take into account the existence of an anisotropy of the electron velocity distribution in the parallel direction. The exponent of the power law of the suprathermal electrons and the fraction of them are typically of the order of $2 \div 3$ and $5-10 \%$, respectively (let us stress that we consider here suprathermal tails produced by any solar wind disturbances which can enhance the hot tail population, as already discussed in the Introduction). No anisotropy between the parallel and the perpendicular temperatures of the electrons of the bulk is introduced. The only anisotropy considered here is due to the existence of the suprathermal tail extending in one direction only. We assume that the ions are described by a maxwellian with a temperature $T_{i}$, as discussed in the previous section.

Figure 1 shows the results of the numerical calculations performed with the values of $\omega_{p}, \omega_{c}, T_{e}$ and $T_{i}$ given by Eq. (45). The electron velocity distribution $f_{0}\left(v_{z}, v_{\perp}\right)$ is represented (see the Fig. 1a) by contour lines of constant level, as a function of the normalized parallel and perpendicular velocities $v_{z} / v_{*}$ and $v_{\perp} / v_{*}$, showing the suprathermal electron tail extending in the parallel velocity direction for $v_{z}>0$. The normalization factor $v_{*}$ is the thermal velocity corresponding to $T_{e}=1 \mathrm{eV}$, that is $v_{*} \simeq 5.910^{5}$ $\mathrm{ms}^{-1}$. Figures $1 \mathrm{~b}$ and $1 \mathrm{c}$ present the contour lines of constant level of the normalized real frequency $\omega_{r} / \omega_{c}$ and the normalized growth rate $\gamma / \omega_{c}$, as a function of the normalized parallel and perpendicular wave numbers $k_{z} v_{*} / \omega_{c}$ and $k_{\perp} v_{*} / \omega_{c}$, respectively. One can see that oblique propagating whistlers are excited through the fan instability $(\gamma>0)$ over a large domain of $k_{\perp}$, which covers the region where such waves were observed (Eq. (45); indeed, values of $k_{\perp}$ given in Eq. (45) correspond to $0.6 \lesssim k_{\perp} v_{*} / \omega_{c} \lesssim 1.2$, that is to normalized growth rate values of the order of $\gamma / \omega_{c} \sim 5$ $10^{-3}$ (see the Fig. 1c). The lower frequency whistlers are excited by the highest energy electrons and inversely (see the Figs. $1 \mathrm{~b}$ and c). At given $k_{\perp}$, the growth rate increases when $k_{z}$ decreases, that is when the electrons in anomalous Doppler resonance with the waves become more and more energetic. One estimates that for $0.01 \lesssim k_{z} v_{*} / \omega_{c} \lesssim 0.1$, what corresponds to electron normalized resonant velocities $10 \lesssim v_{r e s} / v_{*} \simeq \omega_{c} / k_{z} v_{*} \lesssim 100\left(\omega_{r} \ll \omega_{c}\right)$, the waves are driven unstable by electrons with energies ranging from $100 \mathrm{eV}$ to $10 \mathrm{keV}$. This range of energy corresponds well to the region where the hot suprathermal tail of electrons can be formed after the quasi-linear relaxation of the beam (observed to have energies up to $12 \mathrm{keV}$; Eq. 45). Note that by taking the contribution of the resonances $n=0$ and $n=-1$ off the expression of the growth rate, one has checked that the instability is due to the anomalous cyclotron resonance. In conclusion, one possible source of the whistlers observed

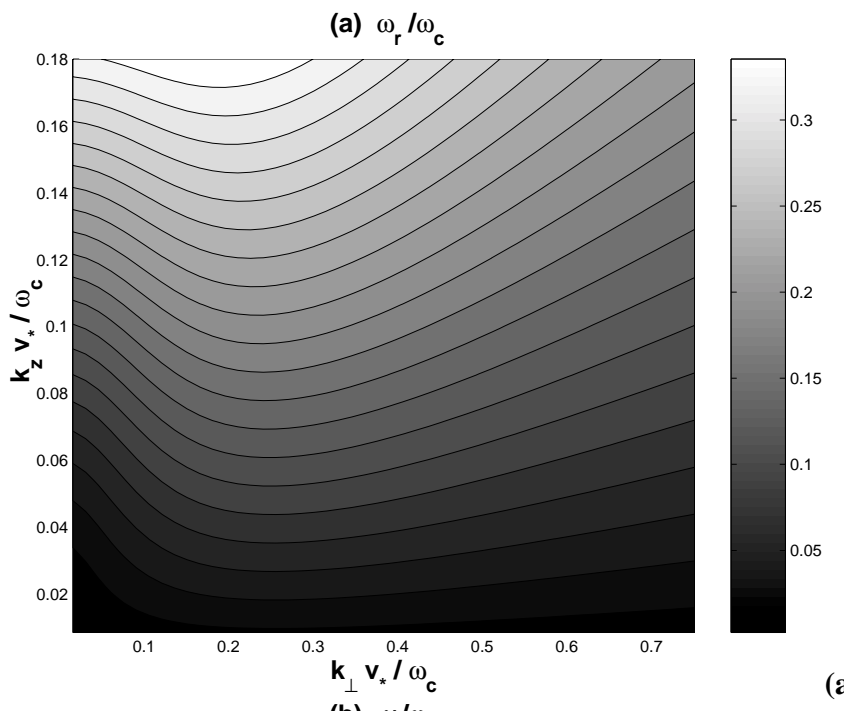

(a)

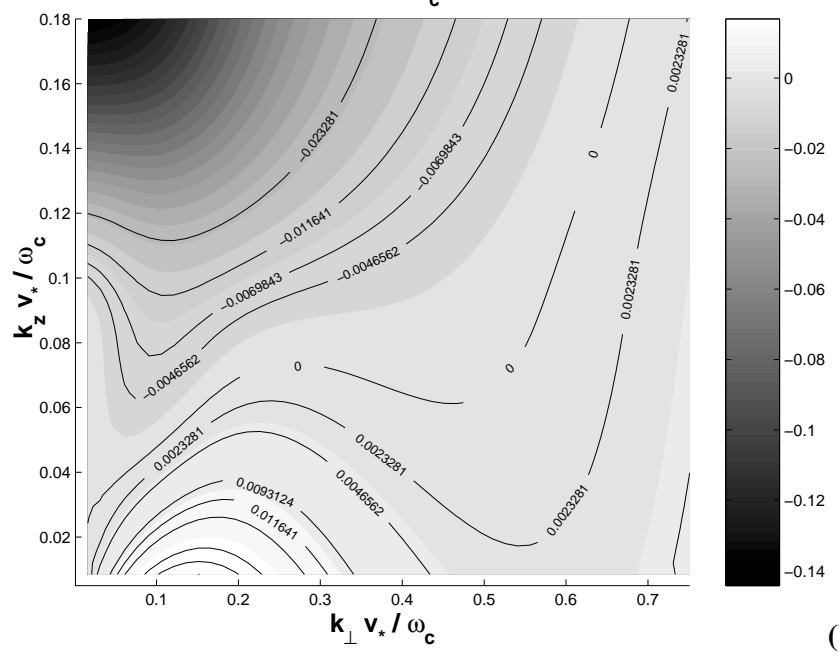

(b)

Fig. 2. Numerical calculations for solar wind plasma conditions: contour lines of constant level of (a) the normalized frequency $\omega_{r} / \omega_{c}$ as a function of the normalized parallel and perpendicular wave numbers $k_{z} v_{*} / \omega_{c}$ and $k_{\perp} v_{*} / \omega_{c}$, and (b) the normalized growth rate $\gamma / \omega_{c}$ as a function of $k_{z} v_{*} / \omega_{c}$ and $k_{\perp} v_{*} / \omega_{c}$. The solar wind parameters are: $\omega_{p} / \omega_{c} \simeq 130, \omega_{c} / 2 \pi \simeq 100 \mathrm{~Hz}$, $\omega_{p} / 2 \pi \simeq 13 \mathrm{kHz}, T_{e} \simeq 15 \mathrm{eV}, T_{e} / T_{i} \simeq 4$. For $(\mathrm{b})$, the values of the calculated growth rates are indicated on the picture.

in Ergun et al. (1998) and Thejappa and MacDowall (1998) is the free energy of the suprathermal electron tail which can excite waves through the fan instability.

Another example can be provided by using the data (Eq. (46) from Thejappa et al. (1995) and Ergun et al. (1998) which are very close to the previous ones; however, in this case, whistlers of larger perpendicular wavelengths have been observed, corresponding to domains of wave numbers where the fan instability is also stronger. Comparing the calculated values of the frequencies $\omega_{r} / \omega_{c}$ and the growth rates $\gamma / \omega_{c}$ of the waves (see Figs. 2a and b) with the measurements of Thejappa et al. (1995), where whistlers with $k_{\perp} \simeq 5.310^{-4} \mathrm{~m}^{-1}\left(k_{\perp} v_{*} / \omega_{c} \simeq 0.5\right)$ and $\omega_{r} / \omega_{c} \simeq 0.38$ 

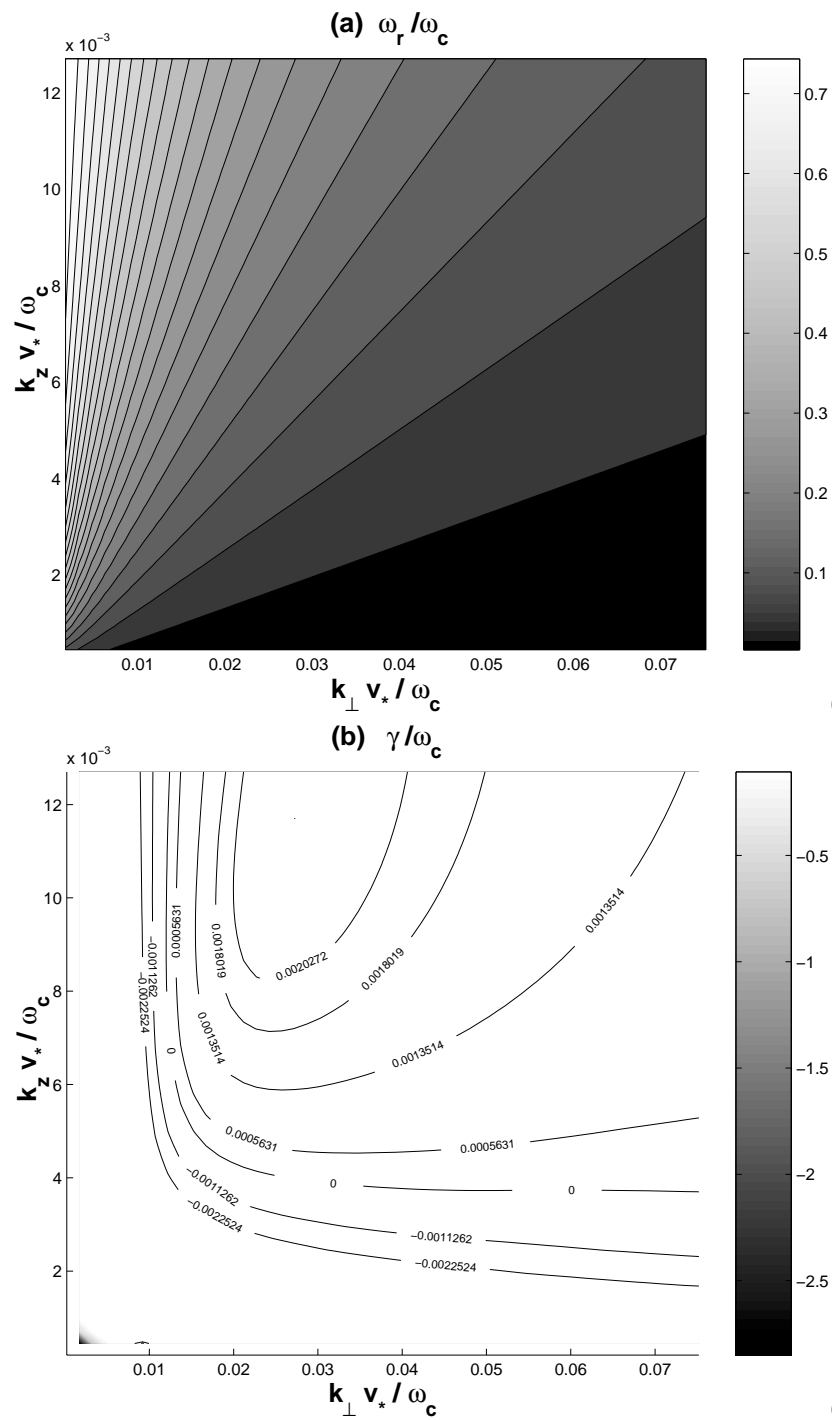

(a)

(b)

Fig. 3. Numerical calculations for auroral plasma conditions: contour lines of constant level of (a) the normalized frequency $\omega_{r} / \omega_{c}$ as a function of the normalized parallel and perpendicular wave numbers $k_{z} v_{*} / \omega_{c}$ and $k_{\perp} v_{*} / \omega_{c}$, and (b) the normalized growth rate $\gamma / \omega_{c}$ as a function of $k_{z} v_{*} / \omega_{c}$ and $k_{\perp} v_{*} / \omega_{c}$. The solar wind parameters are: $\omega_{p} / \omega_{c} \simeq 0.8, \omega_{c} / 2 \pi \simeq 1.15 \mathrm{MHz}, \omega_{p} / 2 \pi \simeq 0.9$ $\mathrm{MHz}, T_{e} \simeq 0.2 \mathrm{eV}$. For (b), the values of the calculated growth rates are indicated on the picture.

were observed, one can see that the domain where the fan instability destabilizes whistlers overlaps the region of observation of such waves (nevertheless, in our case, the waves are excited with similar wave numbers but with lower frequencies). The domain of instability corresponds roughly to $0.01 \lesssim k_{z} v_{*} / \omega_{c} \lesssim 0.06$ (Fig. $2 \mathrm{~b}$ ), that is to $16 \lesssim v_{\text {res }} / v_{*} \simeq$ $\omega_{c} / k_{z} v_{*} \lesssim 100$, that is to resonant energies above $250 \mathrm{eV}$. Finally, the same conclusion can be stated as in the previous paragraph.

Let us now consider the auroral plasma conditions (Eq. (47). In this case, the solar wind parameters, and in particular the ratio $\omega_{p} / \omega_{c}$, are very different from the pre- vious ones. The observed range of wave numbers (Eq. (47) corresponds to the domain $0.01 \lesssim k_{\perp} v_{*} / \omega_{c} \lesssim 0.05$, where the calculations show that sheared whistlers can be driven unstable, as one can see on Figs. 3a and b which represent the contour lines of the constant level of $\omega_{r} / \omega_{c}$ and $\gamma / \omega_{c}$ as a function of $k_{z} v_{*} / \omega_{c}$ and $k_{\perp} v_{*} / \omega_{c}$. Whistlers are destabilized by the fan instability only for $k_{z} v_{*} / \omega_{c} \gtrsim 0.004$, which corresponds to resonant electrons with $v_{\text {res }} / v_{*} \simeq \omega_{c} / k_{z} v_{*} \lesssim 250$, that is to electrons with energies ranging up to $60 \mathrm{keV}$; such electrons were not observed in Ergun et al. (1993) but are known to exist in the disturbed auroral magnetosphere. However, whistlers with higher frequencies than those observed, that is with $0.1 \lesssim \omega_{r} / \omega_{c} \lesssim 0.2$, can be excited through the fan instability by low energy electrons, as those considered by Ergun et al. (1993), but higher energy electrons are needed to excite sheared whistlers in the same ranges of wave numbers and frequencies, as indicated in Eq. (47).

In conclusion, the possibility to excite oblique propagating whistlers or lower hybrid waves by the fan instability in solar wind plasmas has been demonstrated. As during the linear and the nonlinear stages of its evolution, this instability can give rise to characteristic peculiarities in the parallel velocity distribution of the electrons (as bumps in the suprathermal tail, for example), it can indirectly drive other instabilities (as bump-in-tail instabilities generating Langmuir waves) and thus eventually play a fundamental role in the nonlinear wave-wave and wave-particle interaction processes observed in the solar wind in various regions of the interplanetary space and of the near-Earth's environment. Moreover, the nonlinear stage of the fan instability, which will be studied in detail in a forthcoming paper, could influence significantly the nonlinear physical mechanisms and, more specifically, on the Langmuir turbulence processes which govern the solar type III radio bursts generation.

Acknowledgements. The authors acknowledge the Centre National de la Recherche Scientifique (CNRS, PICS 1310, France), the Institut Universitaire de France (Paris), the Russian Academy of Sciences and the Russian Foundation for Basic Research (RFBR, Grant $\mathrm{N}^{\circ}$ 901-05-22003NCNIa) for their financial support.

Topical Editor R. Forsyth thanks G. Mann and another referee for their help in evaluating this paper.

\section{References}

Abalde, J. R., Alves, M. V., and Chian, A. C.-L.: Nonlinear generation of type III solar radio bursts by the hybrid modulational instability, Astron. Astrophys., 331, L21-L24, 1998.

Atamanyuk, B. and Volokitin, A. S.: Suppression of the current instability by decay processes, Plasma Phys. Rep., 27, 7, 598607, 2001.

Bardwell, S. and Goldman, M. V.: Three-dimensional Langmuir wave instabilities in type III solar radio bursts, Astrophys. J., 209, 919, 1976.

Chian, A. C. L. and Abalde, J. R.: Nonlinear coupling of Langmuir waves with whistler waves in the solar wind, Sol. Phys., 184, 403-419, 1999. 
Corotini, F. V., Kennel, C. F., Scarf, F. L., and Smith, E. J.: Whistler mode turbulence in the disturbed solar wind, J. Geophys. Res., 87, A8, 6029-6044, 1982.

Ergun, R. E., Delory, G. T., Klementis, E., Carlson, C. W., McFadden, J. P., and Roth, I.: VLF waves growths from dispersive bursts of field-aligned electron fluxes, J. Geophys. Res., 98, A3, 3777-3787, 1993.

Ergun, R. E., Larson, D., Lin, R. P., Mc Fadden, J. P., Carlson, C. W., Anderson, K. A., Muschietti, I., McCarthy, M., Parks, G. K., Rème, H., Bosqued, J. M., D’Uston, C., Sanderson, T. R., Wenzel, K. P., Kaiser, M., Lepping, R. P., Bale, S. D., Kellogg, P., Bougeret, J. L.: Wind spacecraft observations of solar impulsive electron events associated with solar type III radio bursts, Astrophys. J., 503, 435-445, 1998.

Forslund, D. W., Kindel, J. M., and Lindman, E. L.: Parametric excitation of electromagnetic waves, Phys. Rev. Lett., 29, 249, 1972.

Gary, S. P. and Cairns, I. H.: Electron temperarature anisotropy instabilities: whistler, electrostatic and Z mode, J. Geophys. Res., 104, A9, 19835-19842, 1999.

Gary, S. P., Feldman, W. C., Forslund, D. W., and Montgomery, M. D.: Heat flux instabilities in the solar wind, J. Geophys. Res., 80, 4197-4203, 1975.

Ginzburg, L. and Zheleznyakov, V. V.: On possible mechanisms of sporadic solar radio emission, Sov. Astron., 2, 653, 1958.

Gurnett, D. A. and Anderson, R. R.: Electron plasma oscillations associated with type III radio bursts, Science, 194, 1159, 1976.

Haber, I., Huba, J. D., Palmadesso, P., and Papadopoulos, K.: Slope reversal of a monotonically decreasing electron tail in a strong magnetic field, Phys. Fluids, 21, 6, 1013-1016, 1978.

Hoppe, M. M., Russell, C. T., Eastman, T. E., and Frank, L. A.: Characteristics of the ULF waves associated with upstream ion beams, J. Geophys. Res., 87, A2, 643-650, 1982.

Jie Zhao, Jun-Ichi Sakai, and Nishikawa, K. I.: Excitation of whistler waves driven by an electron temperature anisotropy, Sol. Phys., 168, 2, 345, 1996.

Kadomtsev, B. B. and Pogutse, O. P.: Electric conductivity of a plasma in a strong magnetic field, Soviet Phys. JETP, 26, 1146, 1967.

Kaplan, S. A. and Tsytovich, V. N.: Plasma Astrophysics, Pergamon Press, Oxford, 1973.

Kellogg, P. J., Goetz, K., Howard, R. L., and Monson, S. J.: Evidence for Langmuir wave collapse in the interplanatary plasma, Geophys. Res. Lett., 19, 1303, 1992a.

Kellogg, P. J., Goetz, K., Lin, N., Monson, S. J., Balogh, A., Forsyth, R. J., and Stone, R. G.: Low-frequency magnetic signals associated with Langmuir waves, Geophys. Res. Lett., 19, 12, 1299-1302, 1992b.

Kennel, C. F. and Wong, H. V.: Resonant particle instabilities in a uniform magnetic field, J. Plasma Phys., 1, 75-80, 1967.

Kennel, C. F., Scart, F. L., Corotini, F. V., Fredricks, R. W., Gurnett, D. A., and Smith, E. J.: Correlated whistler and electron plasma oscillations bursts detected on ISEE-3, Geophys. Res. Lett., 7, 129, 1980.

Kuo, S. P. and Lee, M. C.: Parametric excitation of whistler waves by HF heater, J. Atmos. Terr. Phys., 51, 727-731, 1989.

Leyser, T. B.: Parametric interaction between upper-hybrid and lower-hybrid waves in heating experiments, Geophys. Res. Lett., 18, 3, 408, 1991.

Lin, N., Kellogg, P. J., MacDowall, R. J., Scime, E. E., Balogh, A., Forsyth, R. J., McComas, D. J., and Phillips, J. L.: Very low frequency waves in the heliosphere: Ulysses observations, J.
Geophys. Res., 103, A6, 12 023-12 035, 1998.

Lin, R. P., Levedahl, W. K., Lotko, W., Gurnett, D. A., and Scarf, F. L.: Evidence for nonlinear wave-wave interaction in solar type III radio bursts, Astrophys. J., 308, 954-965, 1986.

Lin, R. P., Potter, D. W., Gurnett, D. A., and Scarf, F. L.: Energetic electrons and plasma waves associated with a solar type III radio burst, Astrophys. J., 251, 364-373, 1981.

Luo, Q., Chian, A. C.-L., and Borotto, F. A.: Broadband theory of Langmuir-whistler events in the solar wind, Astron. Astrophys., 348, 1, L13, 2000.

Mace, R. L.: Whistler instability enhanced by suprathermal electrons within the Earth's foreshock, J. Geophys. Res., 103, A7, 14 643-14 654, 1998.

Melrose, D. B.: Comments on "Neutralization and stabilization of particle streams in the corona and type III radio bursts" by D. F. Smith, Solar Phys., 35, 441, 1974.

Mikhailovskii, A. B.: Theory of Plasma Instabilities, Consultants Bureau, New York, 1974.

Moullard, O., Burgess, D., and Bale, S. D.: Whistler waves observed during an in situ solar type III radio bursts, Astron. Astrophys., 335, 703-708, 1998.

Moullard, O., Burgess, D., Salem, C., Mangeney, A., Larson, D. E., and Bale, S. D.: Whistler waves, Langmuir waves and single loss cone electron distribution inside a magnetic cloud: Observations, J. Geophys. Res., 106, A5, 8301-8313, 2001.

Muschietti, L., Roth, I., and Delory, G.: Oblique turbulence driven by field-aligned electron fluxes in the auroral ionosphere, J. Geophys. Res., 102, A12, 27 217-27 226, 1997.

Omelchenko, Yu. A., Shapiro, V. D., Shevchenko, V. I., AshourAbdalla, M., and Schriver, D.: Modified lower hybrid fan instability exited by precipitating auroral electrons, J. Geophys. Res., 99, A4, 5965-5975, 1994.

Papadopoulos, K., Goldstein, N. L., and Smith, R. A.: Stabilisation of electron stream in type III solar radio bursts, Astrophys. J., 190, 175, 1974.

Parks, G. K., McCarthy, M., Fitzenreiter, R. J., Etcheto, J., Anderson, K. A., Eastman, T. E., Frank, L. A., Gurnett, D. A., Huang, C., Lin, R. P., Lui, A. T. Y., Ogilvie, K. W., Pedersen, A., Rème, H., and Williams, D. J.: Particle and field characteristics of the high-latitude plasma sheet boundary layer, J. Geophys. Res., 89, A10, 8885-8906, 1984.

Sawhney, B. K., Tripathi, V. K., and Singh, S. V.: Parametric coupling of a high-frequency electromagnetic wave to whistler and low-hybrid waves in the ionosphere, Phys. Plasmas, 3, 1, 386391, 1996.

Sentman, D. D., Thomsen, M. F., Gary, S. P., Feldman, W. C., and Hoppe, M. M.: The oblique whistler instability in the Earth's foreshock, J. Geophys. Res., 88, A3, 2048-2056, 1983.

Shapiro, V. D. and Shevchenko, V. I.: Quasi-linear theory of relaxation of an electron beam in a magnetoactive plasma, Soviet Phys. JETP, 27, 635-642, 1968.

Sharma, R. P., Atul Kumar, and Raj Kumar: Generation of electrostatic whistler wave by extraordinary EM wave, J. Geophys. Res., 103, 12, 29 505, 1998.

Smith, R. A., Goldstein, M. L., and Papadopoulos, K.: Nonlinear stability of solar type III radio bursts. I. Theory, Astrophys. J., 234, 348, 1979.

Reiner, M. J., Fainberg, J., and Stone, R. G.: Detection of fundamental and harmonic type III radio emission and the associated Langmuir waves at the source region, Astrophys. J., 394, 340, 1992.

Stone, R. G., MacDowall, R. J., Fainberg, J., Hoang, S., Kaiser, 
M. L., Kellogg, P. J., Lin, N., Osherovich, V. A., Bourgert, J. L., Canu, P., Cornilleau-Wehrlin, N., Desch, M. D., Goetz, K., Goldstein, M. L., Harvey, C. C., Lengyel-Frey, D., Manning, R., Reiner, M. J., Steinberg, J. L., and Thejappa, G.: Ulysses radio and plasma wave observations at high southern heliograthic latitudes, Science, 268, 1026-1029, 1995.

Thejappa, G., Wentzel, D. G., and Stone, R. G.: Low-frequency waves associated with Langmuir waves in solar wind, J. Geophys. Res., 100, A3, 3417-3426, 1995.

Thejappa, G. and MacDowall, R. J.: Evidence for strong and weak turbulence processes in the source region of a local type III radio burst, Astrophys. J., 498, 465-478, 1998.

Tokar, R. L., Gurnett, D. A., and Feldman, W. C.: Whistler mode turbulence generated by electron beams in Earth's bow shock, J. Geophys. Res., 89, A1, 105-104, 1984.

Volokitin, A. S. and Lizunov, G. V.: Fan instability of Bernstein modes, Plasma Phys. Rep., 21, 7, 605-609, 1995.
Volokitin, A. S., Krafft, C., and Matthieussent, G.: Whistler waves produced by a modulated electron beam: electromagnetic fields in the linear approach, Phys. Plasmas, 2, 4297, 1995.

Volokitin, A. S. and Krafft, C.: Nonlinear interaction of eleddtron fluxes with low frequency waves, to be submitted to Physics of Plasmas, 2003.

Zaitsev, V. V., Kunilov, M. V., Mityakov, N. A., and Rapoport, V. O.: Generation of type III radio bursts by electron fluxes having a large injection time, Astron. Zh., 50, 252, 1974.

Zaitsev, V. V., Mityakov, N. A., and Rapoport, V. O.: A dynamic theory of type III solar radio bursts, Sol. Phys., 24, 444, 1972.

Zhang, Y. L., Matsumoto, H., and Kojima, H.: Whistler mode waves in the magnetotail, J. Geophys. Res., 104, 12, 28 633-28 644, 1999a.

Zhang, Y. L., Matsumoto, H., Kojima, H., and Omura, Y.: Extremely intense whistler mode waves near the bow shock: Geotail observations, J. Geophys. Res., 104, A1, 449-461, 1999 b. 